\title{
DIETA Y CÁNCER DE PRÓSTATA
}

\section{ROMERO CAGIGAL, A. FERRUELO ALONSO, A. BERENGUER SÁNCHEZ}

Servicio de Urología. Hospital de Getafe. Madrid.

Actas Urol Esp. 27 (6): 399-409, 2003

\section{RESUMEN}

\section{DIETA Y CÁNCER DE PRÓSTATA}

El cáncer de próstata es la neoplasia con mayor incidencia en Estados Unidos y la segunda causa de muerte por cáncer en dicho país. Con unos tratamientos sometidos a debate por su importante tasa de efectos secundarios, sería muy deseable poder establecer mecanismos de prevención que actúen sobre dicha enfermedad, entre estos podrían encontrarse los factores nutricionales.

En el presente trabajo de revisión se abordan los principales factores dietéticos implicados tanto en el desarrollo como en la prevención del cáncer de próstata.

Hacemos especial hincapié en los polifenoles del vino tinto, cuyo influjo sobre la proliferación de células tumorales LNCaP así como sobre la apoptosis ha sido estudiado en nuestro Servicio.

PALABRAS CLAVE: Dieta. Cáncer de próstata. Quimioprevención. Polifenoles.

\section{ABSTRACT}

DIET AND PROSTATE CANCER

Prostate cancer is the first neoplasia in the United States accounting the second in cancer deaths. With all the treatments strategies in debate because of their side effects, is very important try to elucidate prevention mechanisms that may be implicate in the development of this disease, between these, nutrients have been of mayor importance.

In the present review we tried to study the most important nutritional factors implicated in the development and prevention of prostate carcinoma.

We focus our attention over the polyphenols of the red wine, which influence over cellular proliferation and apoptosis in LNCaP cells have been studied in our Department.

KEY WORDS: Diet. Prostate cancer. Chemoprevention. Polyphenols.

$\mathrm{E}^{1}$ cáncer de próstata es el cáncer más frecuentemente diagnosticado entre varones en países desarrollados, y es la segunda causa de muerte por cáncer en dichos países ${ }^{1}$.

En el año 2001 en Estados Unidos se han diagnosticado 1.268.000 nuevos casos de cáncer de los cuales 198.1000 son de próstata, la localización más frecuente es en próstata con un 31\% del total de los tumores ${ }^{2}$.

El cáncer de próstata sigue siendo y probablemente será un problema de salud cada vez mayor con el envejecimiento de la población mundial. El número de hombres con estadios localizados se incrementará en las próximas décadas. Hoy en día, en muchos países occidentales la expectativa de un niño al nacer se acerca a los 80 años $^{3}$. Para el año 2020 se espera un incremento de la población con más de 65 años de edad mayor del $200 \% 4$.

En España, los datos existentes sobre la incidencia y prevalencia son limitados, y presentan cifras notablemente inferiores a las de los grupos americanos, siendo la incidencia de 44,4 por 100.000 varones y año ${ }^{5}$. 
Aunque datos más recientes presentados por Herranz Amo establecen incidencias en la Comunidad de Madrid que se acercan mucho a la que presenta la población blanca en EE.UU, con una incidencia bruta de 100,4 casos por 100.000 hombres $^{6}$. En Getafe (Área Sanitaria número 10 de Madrid) la incidencia media en los últimos diez años es de 40 casos por 100.000 varones y año.

Con unos tratamientos para la enfermedad no exentos de importante morbilidad debemos tratar de dilucidar posibles estrategias de prevención para dicho cáncer. Las características del cáncer de próstata -alta prevalencia, larga latencia, "screening" deficiente y, mortalidad y morbilidad significativas-, lo convierten en un buen candidato para la quimioprevención ${ }^{4}$.

La quimioprevención persigue reducir la probabilidad de desarrollar una enfermedad, administrando una sustancia determinada a individuos sanos $^{3}$. Esta cuando se trata de evitar una enfermedad tumoral, consiste en la prevención o prolongación del comienzo de la carcinogénesis, utilizando agentes para prevenir, suprimir o revertir la transformación maligna.

Los agentes quimiopreventivos se pueden clasificar como bloqueantes o supresores. Los agentes bloqueantes se cree que actúan previniendo el efecto inicial de los agentes carcinogénicos bloqueando su interacción con los receptores diana. Los agentes supresores, sin embargo, suprimirían la evolución y propagación de un proceso neoplásico ya inducido?

La quimioprevención debe realizarse a largo plazo incluso a lo largo de toda la vida, por esta razón, los agentes que se utilicen deben tener mínima toxicidad. La utilización de micronutrientes para esta función, se basa en evidencias de epidemiología humana, resultados de algunos ensayos clínicos, y estudios con modelos animales de carcinogénesis ${ }^{8}$. El agente quimiopreventivo ideal debería ser eficaz, no-tóxico, barato y fácilmente disponible. Este puede formar parte de una dieta modificada, o puede ser sintetizado. Los compuestos dietéticos tienen el beneficio añadido de que con ellos hay gran experiencia, incluyendo amplios estudios epidemiológicos, los cuales ayudan a definir su seguridad y eficacia en humanos ${ }^{9}$. El cáncer de próstata representa un blanco de primer orden para realizar estudios de quimioprevención por sus factores de riesgo conocidos, dependencia hormonal y lesiones precursoras bien establecidas ${ }^{10}$.

Por otro lado, con muchos países en vías de desarrollo adoptando el estilo de vida y los hábitos dietéticos de los países occidentales, la intervención y la educación pueden tener un gran efecto preventivo en la incidencia mundial del cáncer de próstata.

Adlercreutz y colaboradores han establecido que algunos componentes de las dietas mediterránea y asiática protegerían contra el desarrollo del cáncer de próstata, y que la carencia de estos compuestos en la dieta occidental, constituiría un importante factor para el desarrollo de esta enfermedad $^{11}$.

William Fair en los "AUA News" de julio de 1998 se plantea la siguiente cuestión:

"¿Debería considerarse el cáncer de próstata una enfermedad nutricional?"12.Existen hoy en día datos que apuntarían esta posibilidad:

Por un lado la gran discrepancia que existe entre la incidencia de cáncer de próstata microscópico o latente y la enfermedad clínica. La incidencia en cáncer de próstata microfocal es igual en todo el mundo. En un estudio realizado postmorten se objetivó una prevalencia de cáncer de próstata del $3.58 \%, 8.82 \%, 14.28 \%, 23.80 \%$, $31.7 \%$ y $33.3 \%$ en la $3^{a}, 4^{\underline{a}}, 5^{\underline{a}}, 6^{\underline{a}}, 7^{\underline{a}}$ y $8^{\underline{a}}$ décadas respectivamente ${ }^{51}$.Y sin embargo, ¿qué hace que un cáncer microfocal en la mayoría de los hombres no llegue a desarollarse como un cáncer clínico? Probablemente factores ambientales y dentro de estos los que parecen presentar mayor influencia son los nutricionales.

También existen importantes diferencias geográficas en la incidencia de cáncer de próstata a lo largo del mundo. Estas diferencias sugieren que algunos factores dietéticos pueden influir en el desarrollo del cáncer, bien jugando un papel activador o bien como inhibidor de la carcinogénesis ${ }^{13}$. La incidencia del cáncer de próstata en la población negra en USA es 30 superior a la que se da en Osaka (Japón) y 120 veces superior a la Shangai (China).Un interesante estudio observó un aumento entre 3 y 7 veces en la incidencia de cáncer de próstata clínico en la primera generación de Chinos y Japoneses nacidos en 
USA.Dentro de Estados Unidos se ha visto una relación directa entre la ingesta de grasa por persona con la mortalidad por cáncer de próstata, siendo la mortalidad por cáncer de próstata más alta en los estados de USA donde se da el mayor consumo de grasa. La incidencia de cáncer de próstata aumenta en áreas urbanas Japón donde prevalece la dieta occidental.

Ha sido estudiado por diferentes grupos el papel que pueden ejercer ciertas sustancias tanto como protectoras como inductoras del cáncer de próstata. Los principales factores dietéticos que han sido implicados en el desarrollo o prevención de esta enfermedad son los siguientes:

\section{Grasa}

La grasa es el componente dietético que ha sido más relacionado con la incidencia de cáncer de próstata. La dieta en Estados Unidos es rica en grasas en contraposición a la dieta japonesa muy pobre en ellas, país este con una muy baja incidencia de cáncer de próstata.

Numerosos estudios han establecido para los pacientes obesos, un riesgo relativo general de padecer cáncer de próstata de $1,25^{14}$.

En estudios que correlacionaban las tasas internacionales de mortalidad por cáncer de próstata, y el consumo per cápita de grasa se ha visto que el riesgo relativo se eleva a 1,54 en los consumidores de grasa animal ${ }^{15}$.

Sin embargo otros estudios experimentales apuntan a que la grasa de la dieta no influiría en la incidencia de cáncer de próstata, aunque sí la ingesta total de calorías, esta conclusión es apoyada por Albanes en estudios con pacientes $^{16}$.

Los aceites vegetales, por el contrario, no parecen incrementar el riesgo de padecer cáncer de próstata, y los cereales tendrían incluso un papel protector ${ }^{17}$.

\section{Ácidos grasos esenciales}

Los ácidos grasos esenciales, que se derivan de la dieta, al contrario que los no esenciales, que se producen en el organismo, juegan un papel importante en el desarrollo del cáncer de próstata. La concentración de ácido araquidónico y docosapentanóico, ácidos grasos omega-3 (derivados del ácido linolénico), es significativamente inferior en el tejido maligno prostático si lo comparamos con el tejido benigno, mientras que la concentración de ácido oleico es significativamente superior en el tejido maligno que en el benigno ${ }^{18}$.

En pacientes con carcinoma de próstata avanzado se ha objetivado una baja proporción entre ácido esteárico/oleico con un índice medio de saturación de $0,466^{19}$. El ácido linoleico estimula el crecimiento de una línea celular de cáncer de próstata hormonoresistente, mientras que los ácidos grasos omega -3 (derivados del ácido linolénico), docosahexanoico y eicosapentanoico inhiben el crecimiento de esa línea celular ${ }^{20}$.

\section{Licopeno}

El licopeno es el carotenoide más prevalente en la dieta occidental, y el más abundante en el suero humano. Se encuentra fundamentalmente en los tomates y en sus derivados y tiene un importante poder antioxidante ${ }^{16}$.

Aunque los tomates son la principal fuente de licopeno, en el estudio de "Auckland sobre Próstata", el consumo de tomates crudos no se asoció con reducción en el riesgo de padecer cáncer de próstata. Sin embargo, muchos de los derivados del tomate como la salsa de espaguetis, sopa de tomate y salsa "Ketchup" son mejores fuentes de licopeno biodisponible que los tomates frescos ${ }^{21}$.

En un estudio con 14.000 varones "Adventistas del Séptimo día" con alta ingesta de tomates y derivados, se observó una reducción en el riesgo de padecer cáncer de próstata del 35\%, y cáncer de próstata agresivo del $53 \%{ }^{22}$.

En la actualidad el Instituto Nacional del Cáncer está llevando a cabo un estudio fase I, para tratar de comprender mejor el papel del licopeno como agente preventivo frente al cáncer de próstata $^{23}$.

\section{Zanahoria y carotenoides}

Los vegetales verde-amarillos (zanahorias, espinacas, lechuga verde y espárragos verdes) contienen más de $600 \mu \mathrm{g}$ de caroteno/100 g.

En Japón se realizó un estudio de cohortes con 265.118 adultos mayores de 40 años, donde se investigó la relación entre estilo de vida y riesgo de enfermedades. En el mismo se vio que el 
consumo diario de vegetales verde-amarillos protegía frente al cáncer de estomago y próstata, asî como, frente a la enfermedad isquémica cardiaca, arteriosclerosis y cirrosis hepática ${ }^{24}$.

Varones que consumen importantes cantidades de zanahoria tienen un riesgo relativo de padecer cáncer de próstata de sólo el 0,66, siendo esta por lo tanto, un factor protector ${ }^{15}$. Sin embargo, el caroteno tiene muy poca influencia sobre el cáncer de próstata con un riesgo relativo de 0,99 , luego deben existir otros componentes en la zanahoria que actúen como factores protectores $^{24}$.

La luteína, carotenoide que se encuentra en grandes cantidades en las verduras cruciferas y en las hojas verdes podría estar relacionada con el cáncer de próstata. La ingestión de $2000 \mu \mathrm{g}$ o más de luteína asociada a zeaxantina comparada con una ingesta inferior a $800 \mu \mathrm{g}$ tiene un efecto protector contra el cáncer de próstata, con una odds-ratio de $0,68^{25}$.

\section{Vitamina E}

La vitamina agrupa a una serie de compuestos llamados tocoferoles y tocotrienoles. Habitualmente los suplementos de vitamina $\mathrm{E}$ se administran en forma de alfa-tocoferol ${ }^{23}$.

La vitamina $\mathrm{E}$ tiene una potente actividad antioxidante, y también protegería contra el cáncer estimulando funciones inmunitarias, disminuyendo la actividad de la proteína $\mathrm{C}$ kinasa (involucrada en la regulación de la proliferación celular) e induciendo apoptosis. Por otro lado se ha visto que esta inhibe el crecimiento de varias líneas celulares de cáncer de próstata $^{26}$.

El estudio más convincente sobre el papel protector de la vitamina E contra el cáncer de próstata fue, el "Estudio de Prevención de Cáncer por alfa-tocoferol y beta-caroteno (ATBC)". En este estudio, fumadores finlandeses recibieron $50 \mathrm{mg}$ de vitamina $\mathrm{E}$ y presentaron una reducción del $33 \%$ en incidencia de cáncer de próstata y una reducción del $41 \%$ en la mortalidad por dicha enfermedad $^{26}$.

\section{Vitamina D}

El papel fundamental de la vitamina D es actuar sobre el metabolismo óseo y mineral. Sin embargo, metabolitos de la vitamina $\mathrm{D}$ inducen diferenciación e inhiben proliferación celular, entre otras, de líneas celulares de cáncer de próstata $^{27}$.

Existen estudios que indican que la forma activa de la vitamina $\mathrm{D}$, la 1,25 dihidroxicolecalciferol o calcitrol, a dosis bajas estimula el crecimiento de células LNCaP, mientras que a dosis más altas $\left(>10^{-9} \mathrm{~mol} / \mathrm{L}\right)$ inhibe la carcinogénesis prostática $^{28}$.

\section{Vitamina A y retinoides}

Los retinoides, productos derivados de la vitamina A, ejercen una acción in vitro suprimiendo la acción de la $5 \alpha$-reductasa y por tanto de la dihidrotestosterona ${ }^{29}$.

También se han encontrado un menor nivel de receptores retinoicos en el tejido tumoral prostático, probablemente indicando que esto contribuya a la posible malignización del epitelio prostático ${ }^{30}$.

\section{Selenio}

El selenio es un oligoelemento que se encuentra en cereales, carne y pescados. Existe gran variabilidad en cuanto al consumo de selenio en la dieta debido a que su disponibilidad depende, en gran medida, de su concentración en el suelo $^{31}$. El selenio a diferentes dosis, tiene diferentes actividades anticarcinogénicas incluyendo protección antioxidante, freno del metabolismo carcinogénico, estimulación inmunitaria y apoptosis ${ }^{32}$. Clark y colaboradores realizaron un estudio controlado y doble ciego con 1.312 pacientes con historia de carcinoma basal o escamoso de piel, a los cuales se les suministró $200 \mu \mathrm{g}$ de selenio o placebo durante una media de 4,5 años. En los pacientes que tomaron selenio, se objetivó una reducción en la tasa de cáncer de próstata del $66 \%$ en comparación con los del grupo placebo ${ }^{32}$.

\section{Fitoestrógenos}

Los fitoestrógenos o estrógenos de las plantas se dividen en lignanos, flavonoides e isoflavonoides. Varios fitoestrógenos han sido relacionados con una menor incidencia de enfermedades crónicas como cardiopatía isquémica, accidentes cerebrovasculares y asma. También 
se ha visto menor incidencia en algunos cánceres con la utilización de estos compuestos. La ingesta de miricetina causó una menor incidencia en cáncer de próstata $(0.43 ; 0.22,0.86 ; \mathrm{P}=$ $0,002)^{33}$.

Existe una importante diferencia en el consumo de fitoestrógenos entre la sociedad americana y la japonesa debido principalmente al consumo de soja y sus derivados, estos podrían ser uno de los causantes de la diferente incidencia de cáncer de próstata en estos dos paí$\operatorname{ses}^{34}$.

También los varones japoneses presentan cifras muy superiores de estos compuestos en suero, especialmente genisteína, en relación con los varones del Reino Unido (la concentración media en orina en varones japoneses fue de 492,7 nmol/L en comparación con la de los británicos de $33,2 \mathrm{nmol} / \mathrm{L})^{35}$.

Los flavonoides se encuentran en importantes cantidades en muchas frutas, vegetales, cereales y vino ${ }^{13}$.

Los fitoestrógenos tienen funciones muy diversas que podrían influir en su capacidad antitumoral contra el cáncer de próstata:

\section{ACTIVIDAD ESTROGÉNICA:}

Los lignanos de los mamíferos (enterolactona y enterodiol), los isoflavonoides (daizeína, genisteína, coumestrol y ecuol) y los flavonoides (apigerina, kaempferol y naringenina) poseen todos ellos, una actividad estrogénica débil ${ }^{36}$.

En los adultos jóvenes, aproximadamente el 98\% de la testosterona plasmática se encuentra unida a la globulina para la unión de hormonas sexuales (SHBG) y a otras proteínas de transporte. Sólo el $2 \%$ está libre, y esta es la fracción biológicamente activa que difunde pasivamente a las células diana prostáticas. Un incremento en la concentración de SHBG reduciría la fracción de testosterona libre plasmática. Los vegetarianos, japoneses y chinos presentan niveles plasmáticos de SHBG más altos, y por tanto, menor concentración de testosterona libre que los occidentales con dieta libre ${ }^{36,37}$.

\section{INHIBICIÓN DE LA 5 $\alpha$-REDUCTASA:}

Los isoflavonoides y los lignanos inhiben la $5 \alpha$-reductasa y la $17 \alpha$-hidroxiesteroide-deshi- drogenasa. Los niveles de marcadores de la actividad 5á-reductasa son menores en los hombres japoneses jóvenes que en sus homólogos occidentales ${ }^{37}$.

\section{INHIBICIÓN DE LA AROMATASA:}

Dos tercios de los estrógenos en el varón se derivan de la conversión periférica de los esteroides C19 adrenales (dehidroepiandrosterona y androstendiona) por el sistema enzimático de la aromatasa, en el tejido muscular y adiposo. La enterolactona y los flavonoides, luteolina $\mathrm{y}$ kaempferol, disminuyeron la actividad de la aromatasa en preadipocitos humanos ${ }^{38}$.

\section{INHIBICIÓN DE LAS PROTEINKINASAS}

\section{TIROXINA-ESPECÍFICAS:}

Las tiroxina-kinasas son enzimas necesarias para el funcionamiento de numerosos receptores de factores de crecimiento. La fosforilización de la tiroxina desempeña un importante papel en la proliferación celular.

La genisteína ha demostrado ser un inhibidor específico de la actividad de la tiroxina-kinasa y, por tanto, bloquea receptores de factores de crecimiento.

\section{INHIBICIÓN DE LAS TOPOISOMERASAS DEL}

$A D N$ :

Las topoisomerasas son enzimas que alteran la conformación del ADN, y son cruciales para la división celular ${ }^{37}$.

La inhibición de las topoisomerasas es hoy en día un objetivo para el diseño de nuevas drogas anticancerígenas.

La genisteína es un potente inhibidor de estas enzimas. La quercetina, fisetina y morina también inhiben las ADN topoisomerasas I y II, mientras que el kaempferol sólo inhibe la ADN topoisomerasa $\mathrm{II}^{39}$.

Existen nutrientes con un alto contenido en fitoestrógenos entre ellos los más importantes son los siguientes:

\section{Arroz}

$\mathrm{El}$ arroz, importante fuente de isoflavonoides, es un producto muy popular en el noreste de Finlandia, área conocida por la menor incidencia de cáncer de próstata de todo el país ${ }^{40}$. 
En un estudio con japoneses en Hawai, Severson y colaboradores establecieron que un incremento en el consumo de arroz y soja se asociaba con una disminución del riesgo de padecer cáncer de próstata ${ }^{41}$.

También se han realizado estudios inoculando células $\mathrm{LNCaP}$ en ratones alimentados con una dieta rica en arroz, en los cuales no se observó progresión tumoral cuando se comparó con controles. Este efecto desapareció cuando se añadió grasa a la dieta ${ }^{39}$.

\section{Soja}

Un reciente estudio usando el modelo animal de adenocarcinoma de próstata Dunning R3327 mostró que en las ratas alimentadas con pienso de arroz o flúor de soja el tumor crecía mucho más lentamente que los tumores en los animales control ${ }^{40}$.

La genisteína, producto predominante en la soja, es un potente inhibidor de la proteína tiroxina-kinasa y topoisomerasa II, enzimas cruciales para la proliferación celular. También es un inhibidor de la angiogénesis y de numerosas enzimas del metabolismo esteroideo como la aromatasa y la 5á reductasa ${ }^{42}$.

Tanto estudios epidemiológicos como estudios sobre emigrantes, han demostrado correlación entre un incremento de los niveles de isoflavonas (en especial genisteína) en el suero y orina de los varones asiáticos y disminución de la incidencia de cáncer de próstata ${ }^{43}$.

\section{Cereales}

Rose y colaboradores han encontrado un efecto protector de las dietas ricas en cereales contra el cáncer de próstata ${ }^{44}$.

\section{Té verde}

El té es la bebida más consumida en el mundo a excepción del agua. El té verde contiene polifenoles, siendo los flavonoles los más importantes. Dentro de estos se encuentran la epicatequina, epicatequina 3-galato, epigalocatequina y epigalocatequina 3 -galato ${ }^{42}$.

En un estudio en el que se inocularon células LNCaP y PC-3 en ratones atímicos, se vio que tras dos semanas de inyección diaria de $1 \mathrm{mg}$ de epigalocatequina se producía una reducción de ambos tumores entre el $20 \%$ y el $30 \%{ }^{45}$.
La epigalocatequina, inhibió el crecimiento tumoral en células LNCaP y la expresión de los genes regulados por andrógenos y hK2. También se ha visto un importante efecto inhibitorio sobre el promotor del PSA ${ }^{46}$.

\section{DIETA MEDITERRÁNEA Y CÁNCER DE PRÓSTATA}

La dieta mediterránea, considerada hoy en día protectora contra cánceres endocrinos, así como, frente a enfermedades cardiovasculares, tiene un alto contenido de frutas, vegetales y pasta, y también un consumo moderado de vino ${ }^{47}$.

Existen estudios realizados en Italia que demuestran un efecto protector de la fruta fresca y los vegetales crudos frente a numerosos cánceres, incluyendo el de próstata ${ }^{48,49}$.

$\mathrm{El}$ vino contiene más de 500 compuestos diferentes: azúcares, ácidos, glicerol, polifenoles, aldehídos, vitaminas, etc. Los polifenoles están presentes en cantidades importantes en vinos tintos, y en menor medida, en los blancos, y contribuyen en gran parte a las cualidades deseables del vino aunque no tienen valor nutritivo.

Estos polifenoles, protegen contra enfermedades cardiovasculares y algunos estudios indican que posiblemente contra el cáncer.

Polifenoles presentes en el vino, en el rango pico y nanomolar, disminuyen la proliferación de líneas celulares hormono sensibles ${ }^{50}$.

Estudios con ratones transgénicos alimentados con catequina, polifenol presente en el vino, observaron un significativo retraso en la aparición del tumor prostático con relación al control.

\section{POLIFENOLES DEL VINO TINTO Y CÁNCER DE PRÓSTATA}

Nosotros hemos estudiamos in vitro la influencia de 5 polifenoles presentes en el vino tinto sobre la proliferación de las células LNCaP de cáncer de próstata humano. Los polifenoles utilizados han sido: quercetina, rutina, morina, ácido gálico y ácido tánico. La quercetina, rutina y morina son flavonoides de la familia de los flavonoles, mientras que ácido gálico es un hidroxibenzoato y el ácido tánico es un tanino (polímero de 8 monómeros de ácido gálico y glucosa).

Para los estudios sobre la proliferación celular y la apoptosis, realizamos la siembra celular en 
placas de 96 pocillos de $1 \mathrm{ml}$ de capacidad con 500 células por pocillo, utilizando medio RPMI 1640 , conteniendo un $10 \%$ de suero fetal bovino (FCS), L-glutamina y antibióticos. Tras la adición de los polifenoles antes descritos, las células se cultivaron durante 4 días $(0,24,48,72$ y 96 horas) al cabo de las cuales se procedió, al procesamiento de las muestras para el estudio de la proliferación celular utilizando un kit específico (Cell Proliferation ELISA BrdU - Roche) y para la evaluación de la apoptosis con otro de detección de muerte celular por ELISA (Cell Death Detection ELISAPLUS, Roche).

Obtuvimos una inhibición significativa de la proliferación celular a las 24 horas de incubación con rutina $(50 \mu \mathrm{M}$ y $75 \mu \mathrm{M})$, morina (75 $\mu \mathrm{M})$, ácido tánico $(10 \mu \mathrm{M}) \mathrm{y}$ ácido gálico (10 $\mu \mathrm{M})$. A las 48 horas con rutina $75 \mu \mathrm{M}$, morina $50 \mu \mathrm{M}$, y ácidos tánico y gálico a $10 \mu \mathrm{M}$. A las 72 horas con quercetina $(10 \mu \mathrm{M})$, rutina $(75$ $\mu \mathrm{M})$, ácido tánico $(5 \mu \mathrm{M})$, y ácido gálico $(10 \mu \mathrm{M})$. A las 96 horas con quercetina $(5 \mu \mathrm{M}$ y $10 \mu \mathrm{M})$, rutina $(50 \mu \mathrm{M}$ y $75 \mu \mathrm{M})$, morina $(50 \mu \mathrm{M}$ y 75 $\mu \mathrm{M})$, ácidos tánico y gálico $(5 \mu \mathrm{M}$ y $10 \mu \mathrm{M})$ representamos la inhibición de la proliferación de las células LNCaP inducida por los polifenoles a lo largo de las 96 horas del experimento con las diferentes dosis de cada nutriente en las Figuras 1, 2 y 3.

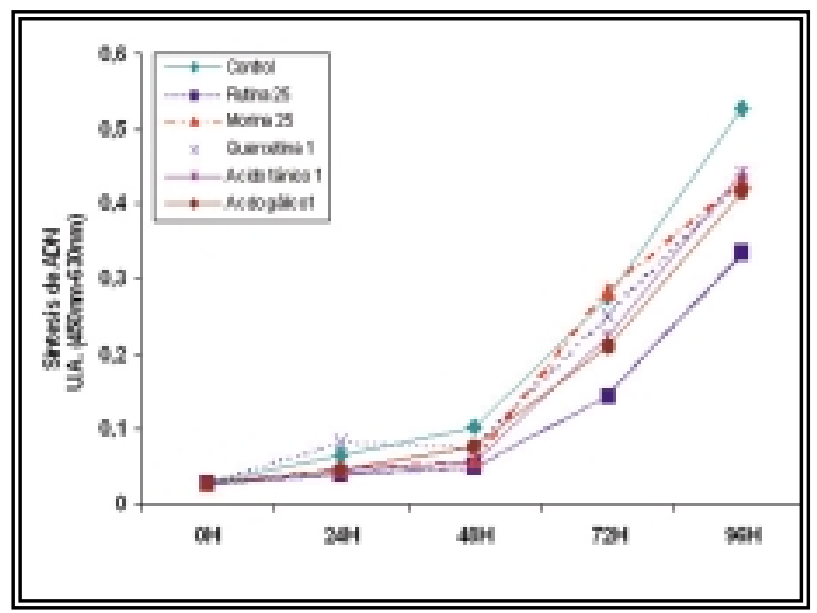

FIGURA 1. Efecto de rutina $25 \mu M$, morina $25 \mu M$, quercetina $1 \mu M$, ácidos gálico $1 \mu M$ y tánico $1 \mu M$ sobre la proliferación de células LNCaP in vitro comparado con control a las 24, 48, 72 y 96 horas. La absorbancia (U.A.) expresa la sintesis de ADN (una forma directa de establecer la proliferación celular). Los resultados se obtuvieron de la media de tres experimentos diferentes.

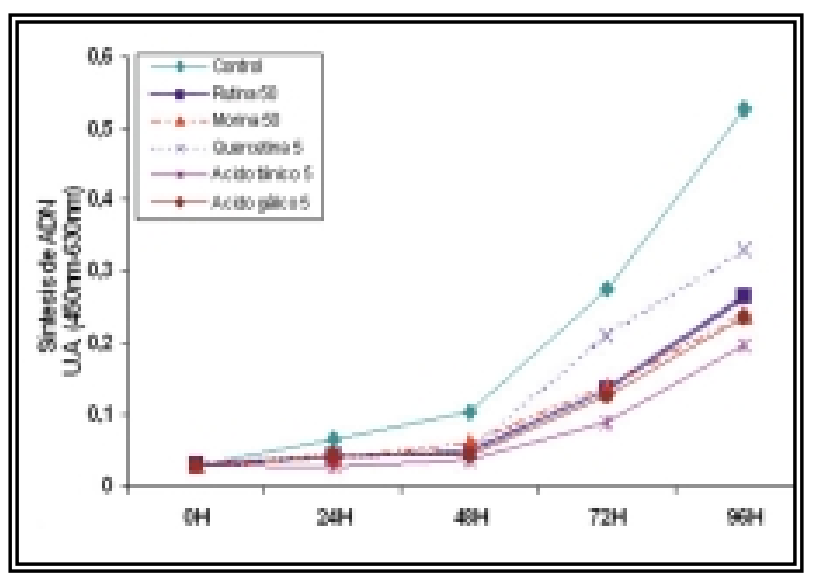

FIGURA 2. Efecto de rutina $50 \mu M$, morina $50 \mu M$, quercetina $5 \mu M$, ácidos gálico $5 \mu M$ y tánico $5 \mu M$ sobre la proliferación de células $L N C a P$ in vitro comparado con control a las 24, 48, 72 y 96 horas. La absorbancia (U.A.) expresa la sintesis de ADN (una forma directa de establecer la proliferación celular). Los resultados se obtuvieron de la media de tres experimentos diferentes.

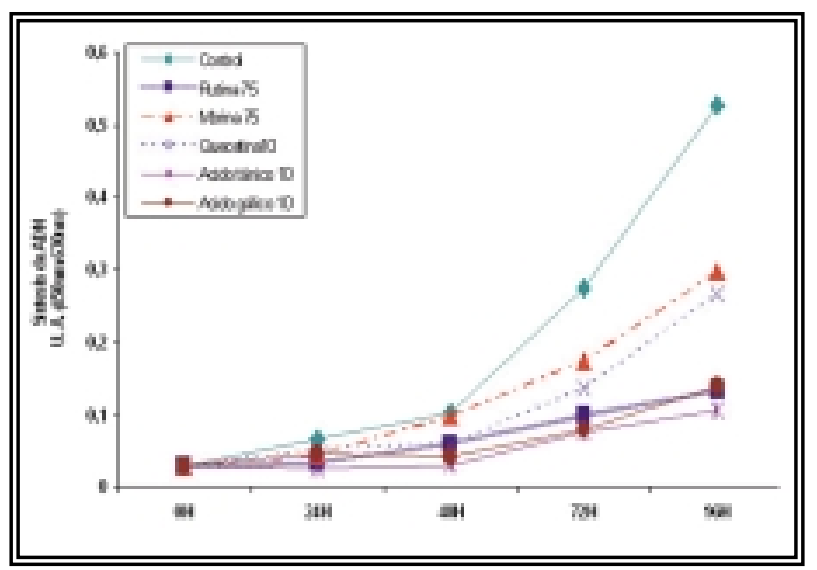

FIGURA 3. Efecto de rutina $75 \mu M$, morina $75 \mu M$, quercetina $10 \mu \mathrm{M}$, ácidos gálico $10 \mu \mathrm{M}$ y tánico $10 \mu \mathrm{M}$ sobre la proliferación de células LNCaP in vitro comparado con control a las 24, 48, 72 y 96 horas. La absorbancia (U.A.) expresa la sintesis de ADN (una forma directa de establecer la proliferación celular). Los resultados se obtuvieron de la media de tres experimentos diferentes.

Las Figuras 4, 5 y 6 son fotos de microscopía óptica a 20 aumentos, en las que observamos la diferente densidad celular tras la inhibición de la proliferación celular inducida por ácido tánico a concentración de $10 \mu \mathrm{M}$ a las 48 y 72 horas. (Figs. 4, 5 y 6).

En cuanto a la inducción apoptótica a las 24 horas no se produjo inducción significativa de apoptosis con ningún nutriente utilizando las dosis que producian inhibición significativa de la proliferación. A partir de las 48 horas de 


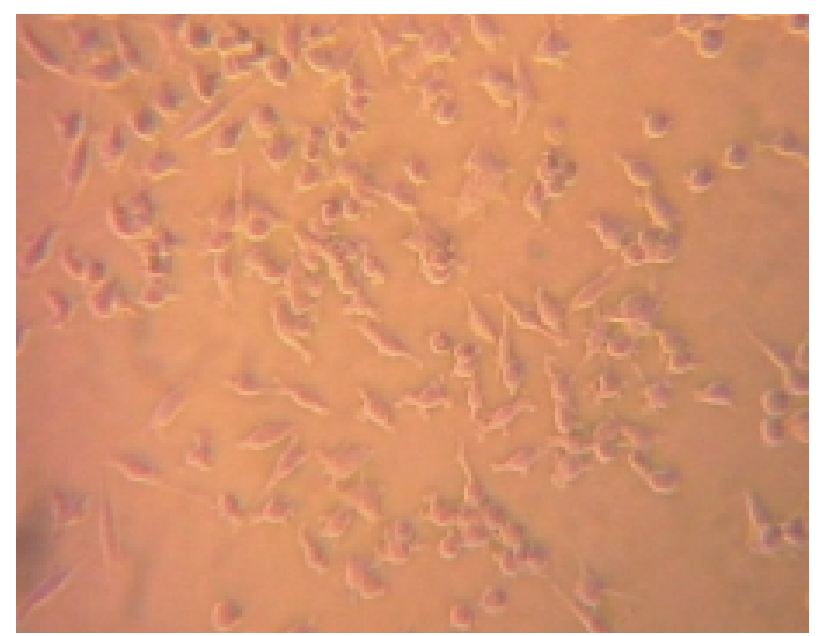

FIGURA 4. Células LnCap en medio de cultivo RPMI-1640 con $5 \%$ FCS. Aspecto de las placas de cultivo a las 0 horas de incubación. Microscopia óptica 20x.

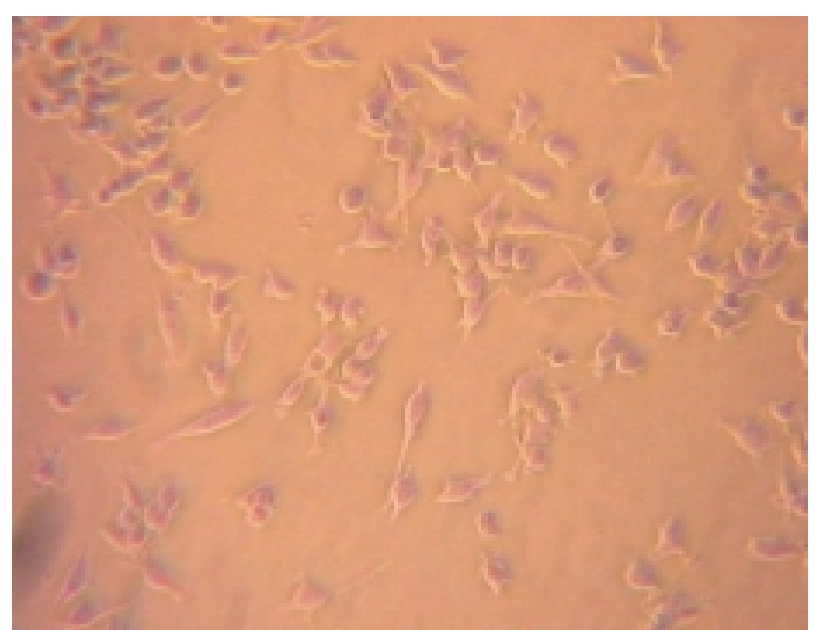

FIGURA 5. Células LnCap en medio de cultivo RPMI-1640 con $5 \%$ FCS. Aspecto de las placas de cultivo tras 48 horas de

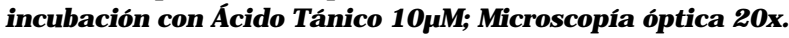

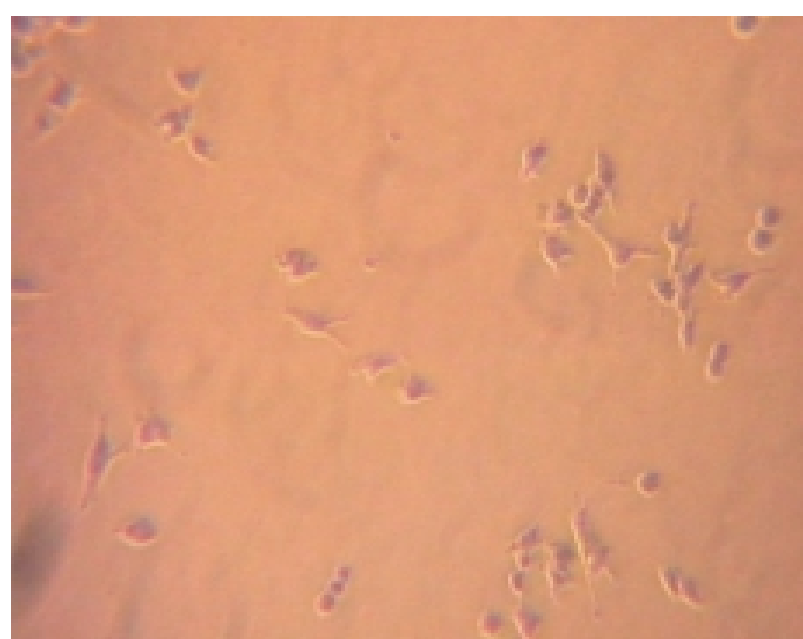

FIGURA 6. Células LnCap en medio de cultivo RPMI-1640 con $5 \%$ FCS. Aspecto de las placas de cultivo tras 96 horas de

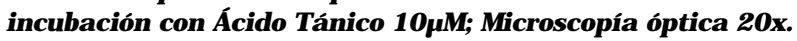

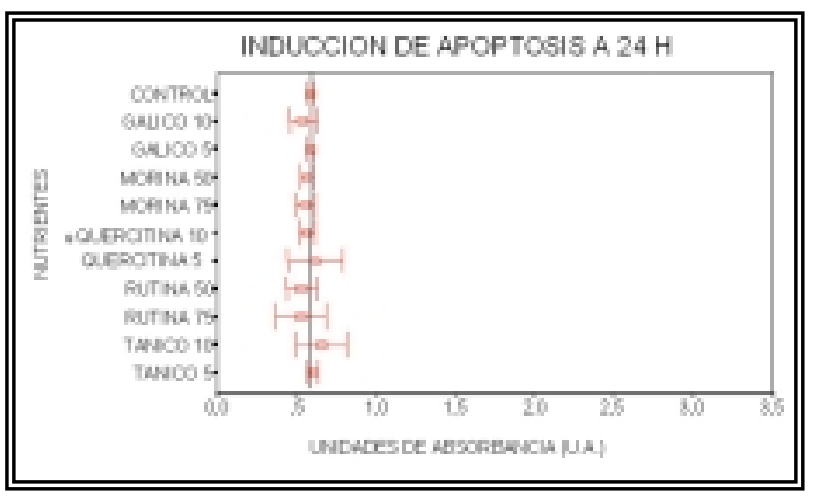

FIGURA 7. ANOVA de la inducción apoptótica por ácido gálico (5 y $10 \mu \mathrm{M}$ ), morina (50 y $75 \mu \mathrm{M})$, quercetina (5 y $10 \mu \mathrm{M}$ ), rutina (50 y $75 \mu \mathrm{M})$ y ácido tánico (5 y $10 \mu \mathrm{M})$, comparada con el control tras 24 horas de incubación. La linea vertical representa la media de los 9 resultados del control.

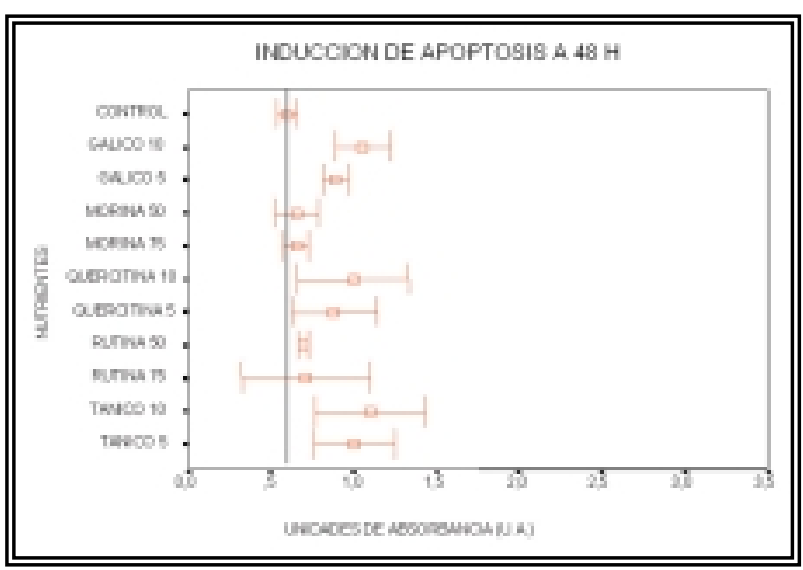

FIGURA 8. ANOVA de la inducción apoptótica por ácido gálico (5 y $10 \mu \mathrm{M})$, morina (50 y $75 \mu \mathrm{M})$, quercetina (5 y $10 \mu \mathrm{M})$, rutina (50 y $75 \mu \mathrm{M})$ y ácido tánico (5 y $10 \mu \mathrm{M})$, comparada con el control tras 48 horas de incubación. La linea vertical representa la media de los 9 resultados del control.

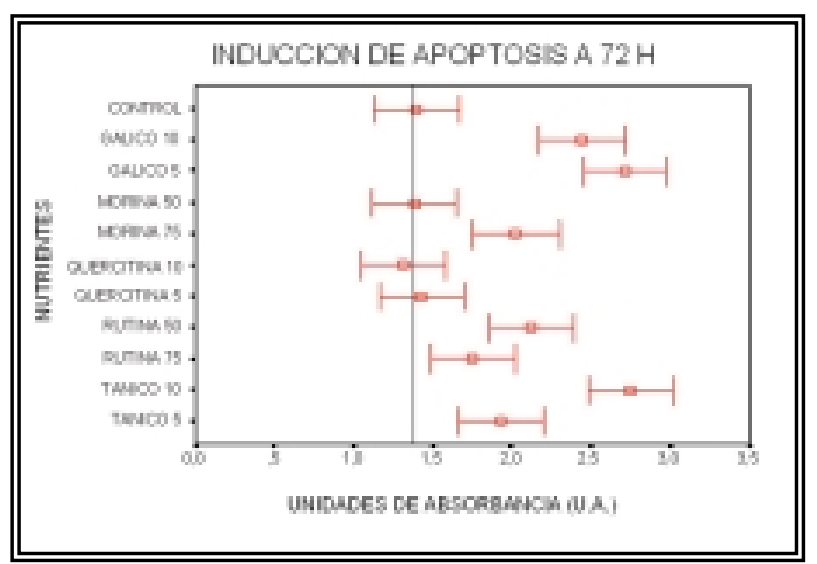

FIGURA 9. ANOVA de la inducción apoptótica por ácido gálico (5 y $10 \mu \mathrm{M})$, morina (50 y $75 \mu \mathrm{M})$, quercetina (5 y $10 \mu \mathrm{M}$ ), rutina (50 y $75 \mu M)$ y ácido tánico (5 y $10 \mu M)$, comparada con el control tras 72 horas de incubación. La linea vertical representa la media de los 9 resultados del control. 
incubación índices apoptóticos significativamente más altos $(\mathrm{p}<0,01)$ se obtuvieron en presencia de ácido gálico 5 y $10 \mu \mathrm{M}$, tánico 5 y $10 \mu \mathrm{M}, \mathrm{y}$ rutina $75 \mu \mathrm{M}$, ( $\mathrm{p}<0,05$ comparados con el control). Este efecto se mantuvo durante las primeras 72 horas. El efecto de la morina $75 \mu \mathrm{M}$, aunque significativo $(\mathrm{p}<0,01)$, sólo apareció a las 72 horas. Inversamente, quercetina 5 y $10 \mu \mathrm{M}$ indujeron apoptosis significativa $(\mathrm{p}<0,05)$ durante las primeras 48 horas, comparada con el control. Los resultados se expresan como un factor de enriquecimiento (EF), en mono y oligonucleosomas, el cual resultará del cociente entre la absorbancia de la muestra (células tratadas con los nutrientes) y la absorbancia del control (células no tratadas), cuanto mayor sea el factor de enriquecimiento, mayor será el grado de muerte celular.

Las Figuras 7, 8 y 9 representan ANOVAS de la inducción apoptótica mediada por los polifenoles en comparación con el control. (Figs. 7, 8 y 9).

Las Figuras 10, 11 y 12 son fotos de microscopía óptica a 20 aumentos, en las que observamos la diferente densidad celular tras la inducción apoptótica por ácido gálico a concentración de $10 \mu \mathrm{M}$ a las 48 y 72 horas. (Figs. 10, 11 y 12).

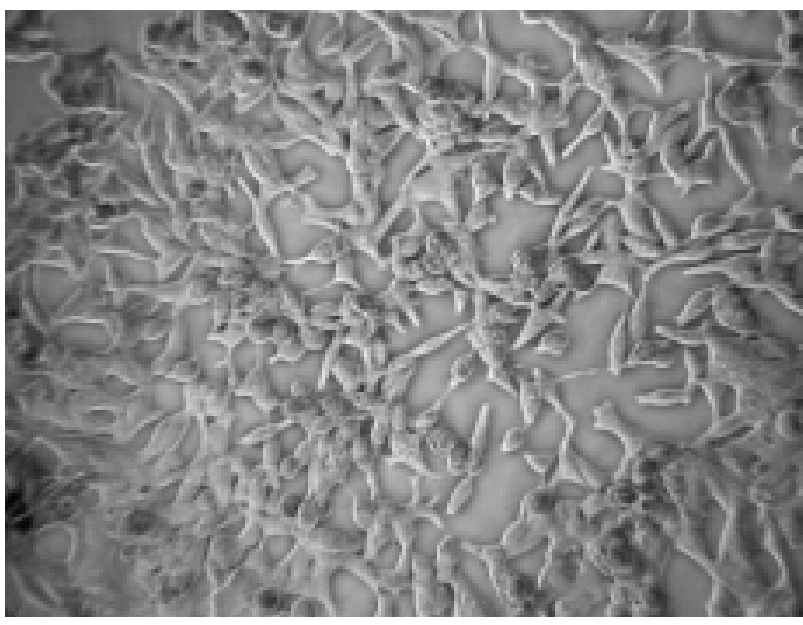

FIGURA 10. Células LnCap en medio de cultivo RPMI1640 con $5 \%$ FCS. Aspecto de las placas de cultivo a las o horas de la siembra; Microscopia óptica 20x.

\section{REFERENCIAS}

1. PATHAK SK, SHARMA RA, MELLON JK.: Chemoprevention of prostate cancer by diet-derived antioxidant agents and hormonal manipulation. Int J Oncol (Review) 2003; 22 (1): 5-13.

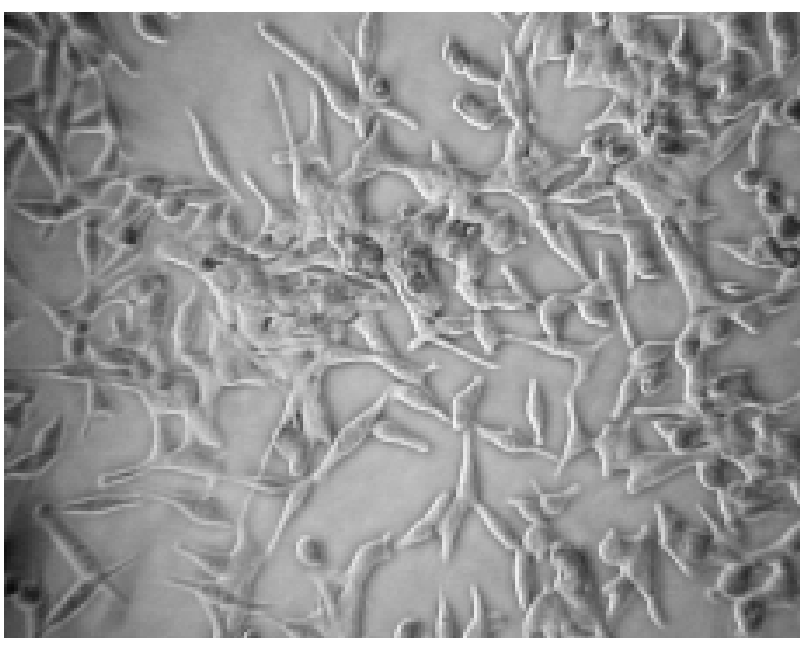

FIGURA 11. Células LnCap en medio de cultivo RPMI-1640 con $5 \%$ FCS. Aspecto de las placas de cultivo a las 48 horas de la siembra, tras incubación con ácido gálico 10 $\mu$ M; Microscopia óptica 20x.

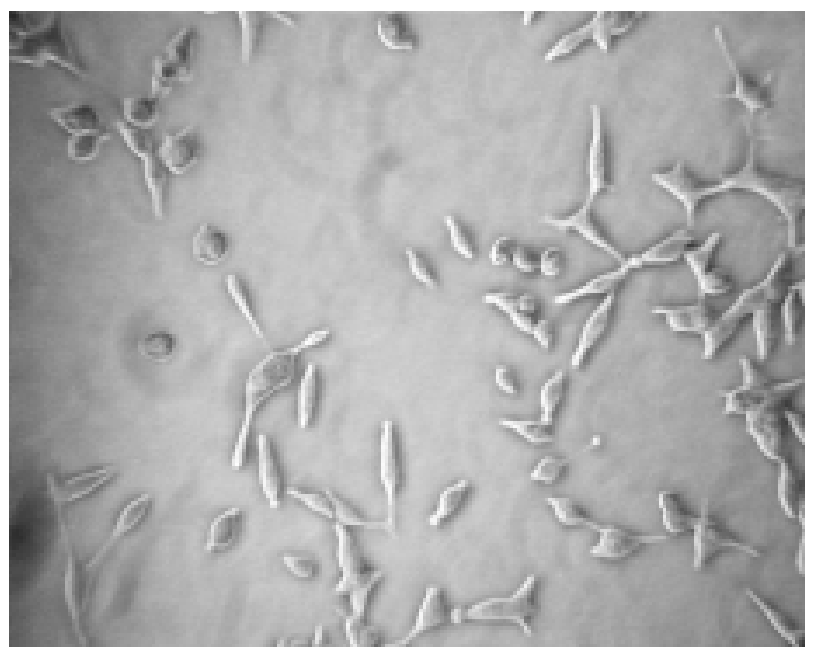

FIGURA 12. Células LnCap en medio de cultivo RPMI-1640 con 5\% FCS. Aspecto de las placas de cultivo a las 72 horas de la siembra, tras incubación con ácido gálico 10 $\mu M$; Microscopia óptica 20x.

2. GREENLEE RT, HILL-HARMON MB, MUTTAY T, THUN M.: Cancer Statistics, 2001: CA Cancer J Clin 2001; 51: 15-36.

3. KIRBY R, FITZPATRICK J, KIRBY M, FITZPATRICK A.: A shared care for prostatic diseases. ISIS Medical Media. 1994.

4. BOYLE P, SEVERI G.: Epidemiology of prostate cancer. Chemoprevention. Eur Urol 1999; 35: 370-376.

5. ARRIZABALAGA M, NAVARRO M, MORAS J y cols.: Incidencia de los principales carcinomas urológicos en el área sanitaria VII de Madrid. Actas Urológicas Españolas 1994: 145: 161.

6. HERRANZ AMO F.: El cáncer de próstata en la Comunidad de Madrid en el año 2000. 2002. 
7. PIERPOINT WS.: Flavonoids in the human diet. En V Cody, E Middleton Jr \& J B Harbone (Eds), Progress in Clinical and Biological Research: 125140. New York: Alan R Liss.

8. BERTRAM FJ, KOLONEL LN, MEYSKENS FL.: Strategies and rationale for chemoprevention in cancer in humans. Cancer Res 1987; 47: 3012-3031.

9. KAMAT AM, LAMM DL.: Chemoprevention of urological cancer. J Urol 1999; 161: 1748-1760.

10. SCHULMAN CC, KELLOFF GJ.: Introduction: strategies for chemoprevention of prostate cancer symposium proceedings. Brussels. Eur Urol 1999; 35: 341-547.

11. ADLERCREUTZ H.: Western diets and Western diseases: some hormonal and biochemical mechanisms and associations. Scan $J$ Clin Lab Invest 1990; 50 (sup. 201): 3-23.

12. FAIR W.: AUA News July 1998.

13. DENIS L, MORTON MS, GRIFFITHS K.: Diet and its preventive role in prostatic disease. Eur Urol 1999; 35: 377-387.

14. ALBANES.: Caloric intake, body weight and cancer. A review. Nutr Cancer 1987; 9: 199-217.

15. KOLONEL LN.: Nutrition and prostate cancer. Cancer Causes Control 1996; 7: 83-94.

16. NGUYEN ML, SCHWARTZ SJ.: Lycopene: chemical and biological properties. Food Thenol 1999; 53: 38-45.

17. GIOVANNUCCI E.: Tomatoes, tomato-base products, lycopene and cancer: review of the epidemiological literature. J Natl Cancer Inst 1999; 91: 317-331.

18. CHAUDRY A, MC CLINTON S, MOFFAT LE, WAHLE KW.: Essential fatty acid distribution in the plasma and tissue phospholipids of patients with benign and malignant prostatic disease. Br J Cancer 1991; 64 (6): 1157-1160.

19. PERSAD RA, GILLATT DA, HEINEMANN D, HABIB NA, SMITH PJ.: Erythrocyte stearic to oleic acid ratio in prostatic carcinoma. Br J Urol 1990; 65 (3): 268-270.

20. ROSE DP, CONNELLY JM.: Effects of fatty acids and prostate cancer. Lipids 1992; 27: 798-803.

21. HIRAYAMA T.: A large-scale cohort study on cancer risks by diet - with special reference to the riskreducing effects of green-yellow vegetable consumption. En Hayash Y et al. (Editores); Diet, Nutrition and Cancer. Utrecht, VNU Sci Press/ Tokyo, Jpn Sci Soc Press 1986: 41-53.

22. COHEN HJ, CRISTAL AR, STANFORD JL.: Fruit and vegetables ingestion, and prostate cancer risk. $J$ Natl Cancer Inst 2000; 92: 61-68.

23. KUMAR NB, BESTERMAN KB.: Nutrients in the chemoprevention of prostate cancer: current and future prospects. Cancer control; JMCC 1999; 6 (6): $580-586$

24. NORRISH AE, JACKSON RT, SHARPE SJ y cols.: Prostate cancer and dietary carotenoids. Am J Epidemiol 2000; 151: 123-129.

25. HEINONEN OP, ALBANES D, VIRTAMO J y cols. Prostate cancer and supplementation with alphatocopherol and beta-carotene: incidence and mortality in a controlled trial. J Natl Cancer Inst 1998; 90: $440-446$.
26. INSTITUTE OF MEDICINE, NATIONAL ACADEMY OF SCIENCES; Food and nutrition board; panel on dietary antioxidants and related compounds. Dietary reference intakes for vitamin C, vitamin E, selenium and carotenoids: Washington DC: National Academy Press 2000.

27. KONETY BR, JOHNSON CS, TRUMP DL, GETZENBERG RH.: Vitamin D in the prevention and treatment of prostate cancer. Sem Urol Oncol 1999; 17 (2): $77-84$

28. PEELH DM y cols.: Antiproliferative effects of 1,25dihydroxyvitamin $\mathrm{D}_{3}$ on primary cultures of human prostatic cells. Cancer Res 1994; 54: 805-810.

29. HALGUNSET J, SUNDE A, LUNDMO PI: Retinoic acid (AA): an inhibitor of 5-alpha-reductase in Luman prostatic cancer cells. J Steroid Biochem 1987; 28: 731-738.

30. PASQUALI D, THALLER C, EICHELE G.: Abnormal level of retinoic acid in prostate cancer tissues. J Clin Endocrinol Metab 1996; 81: 2186 2191.

31. COMBS GF, GRAY WP.: Chemopreventive agents: selenium. Pharmacol Ther 1998; 79: 179-192.

32. CLARK LC, COMBS GF JR, TURNBULL BW y cols.: Effects of selenium supplementation for cancer prevention in patients with carcinoma of the skin. A randomised controlled trial. Nutritional prevention of cancer study group. JAMA 1996; 276: $1957-$ 1963.

33. KNEKT P, KUMPULAINEN J, JARVINEN R, RISSANEN H, HELIOVAARA M, REUNANEN A, HAKULINEN T, AROMAA A.: Flavonoid intake and risk of chronic diseases. Am J Clin Nutr 2002; 76 (3): 560568.

34. MIKSICEK RJ.: Estrogenic flavonoids: structural requirements for biological activity. Pro Soc Exp Biol Med 1995; 208: 440-450.

35. MORTON MS, ARISAKA O, MIYAKE N, MORGAN LD, EVANS BA.: Phytoestrogen concentrations in serum from Japanese men and women over forty years of age. $J$ Nutr 2002; 132 (10): 3168-3171.

36. ADLERCREUTZ H, BANNWART C, WAHALA $\mathrm{K} y$ cols.: Inhibition of human aromatase by mammalian lignans and isoflavonoid phytoestrogens. $J$ Steroid Biochem Molec Biol 1993; 44: 147-153.

37. CONSTANTINOU A, MEHTA R, RUNYANN C, RAO K, VAUGHAN A, MOON R.: Flavonoids as DNA topoisomerase antagonists and poisons: structureactivity relationships. J Nat Prod 1995; 58: 217 225.

38. KLEEMOLA P, VIRTANEN M, PIETINEN P.: The 1992 diet survey of Finnish adults, vol B2/1994. Helsinki, Helsinki National Public Health Institute, Department of Nutrition. 1994.

39. BYLUND A, ZHANG JX, BERGH A, DAMBER JE y cols.: Rye bran and soy protein delay growth and increase apoptosis of human LNCaP prostate adenocarcinoma in nude mice. Cancer Res 1990; 51 : 847-850.

40. LANDSTROM M, ZHANG JX, HALLMANS G, AMAN P y cols.: Inhibitory effects of soy and rye diets on the development of Dunning R3327 prostate adenocarcinoma in rats. Prostate 1998; 36: 151-161. 
41. SEVERSON RK, NOMURA AM, GROVE AS, STEMMERMANN GN.: A prospective study of demographics, diet and prostate cancer among men of Japanese ancestry in Hawaii. Cancer Res 1989; 49: 1857-1860.

42. GUPTA S, AHMAD N, MUKHTAR H.: Prostate cancer chemoprevention by green tea. Sem Urol Oncol 1999; 17 (2): 70-76.

43. GRIFFITHS K, MORTON MS, DENNIS L.: Certain aspects of molecular endocrinology that relate to the influence of dietary factors on the pathogenesis of prostate cancer. Eur Urol 1999; 35: 443-455.

44. ROSE DP, BOYAR AP, WYNDER EL.: International comparisons of mortality rates for cancer of the breast, ovary, prostate and colon and per capita food consumption. Cancer 1986; 58: 2363-2371.

45. LIAO S, UMEKITA Y, GUO J, KOKONTYIS JM, HIIPAKKA RA.: Growth inhibition and regression of human prostate and breast tumours in athymic mice by tea epigallocathechin gallato. Cancer Lett 1995; 96: 239-243.

46. REN F, ZHANG S, MITCHELL SH, BUTLER R, YOUNG CY.: Tea polyphenols down-regulate the expression of the androgen receptor in LNCaP prostate cancer cells. Oncogene 2000 6; 19 (15): 19241932.

47. BLOCK G, PATTERSON B, SUBAR A.: Fruit, vegetables and cancer prevention: a review of the epidemiological evidence. Nutr Cancer 1992; 18: 1-29.
48. NEGRI E, LA VECCHIA C, FRANCESCHI S, D'AVANZO B, PARAZZINI F.: The role of vegetables and fruit in cancer risk; en Hill MJ, Giacosa A, Caygill CPJ (editores): Epidemiology of diet and cancer 1994. Chichester, Horwood: 327-334.

49. LA VECCHIA C, DE CARLI A, NEGRI E, PARAZZINI F.: Epidemiological aspects of diet and cancer: a summary review of case-control studies from Northern Italy. Oncology 1988; 45: 364-370.

50. DAMIANAKI A, BAKOGEORGOU E, KAMPA M, NOTAS G, HATZOGLOU A, PANAGIOTOU S, GEMETZI C, KOUROUMALIS E, MARTIN PM, CASTANAS.: Potent inhibitory action of red wine polyphenols on human breast cancer cells. Cell Biochem 2000 6; 78 (3): 429-441.

51. SÁNCHEZ-CHAPADO M, OLMEDILLA G, CABEZA M, DONAT E, RUIZ A.: Prevalence of prostate cancer and prostatic intraepithelial neoplasia in caucasian Mediterranean males: an autopsy sudy. Prostate 2003, 15; 54 (3): 238-247.

Dr. I. Romero Cagigal

Servicio de Urología. Hospital de Getafe

Ctra. de Toledo km. 12,500

28905 Getafe (Madrid)

(Trabajo recibido el 24 marzo de 2003) 